(1)

George Fox

UNIVERSITY
Digital Commons @ George Fox University

Faculty Publications - School of Physical

Therapy

School of Physical Therapy

4-1-2007

The Relationship Between Ankle, Hindfoot, and Forefoot Position and Posterior Tibial Muscle Excursion

Adolph S. Flemister

Christopher G. Neville

Jeff Houck

Follow this and additional works at: https://digitalcommons.georgefox.edu/pt_fac

Part of the Physical Therapy Commons 


\title{
The Relationship Between Ankle, Hindfoot, and Forefoot Position and Posterior Tibial Muscle Excursion
}

\author{
Adolph S. Flemister, M.D. ${ }^{1}$; Christopher G. Neville, M.D. ${ }^{2,3}$; Jeff Houck, P.T.; Ph.D. ${ }^{3}$
}

Rochester, $N Y$

\begin{abstract}
Background: The purpose of this study was to examine the relationship of forefoot position in the transverse plane (abduction/adduction), hindfoot position in the frontal plane (eversion/inversion), and ankle position in the sagittal plane (plantarflexion/dorsiflexion) with posterior tibialis (PT) muscle excursion using an in vitro cadaver model. Methods: Seven freshfrozen cadaver specimens were potted and mounted on a frame. The PT tendon was dissected $15 \mathrm{~cm}$ proximal to the medial malleolus, and a $5-\mathrm{kg}$ weight was sutured to the tendon. A six-camera motion analysis system (Optotrak, Northern Digital, Inc.) was used to track three-dimensional (3D) motion of the tibia, calcaneus (hindfoot) and first metatarsal (forefoot) using bone pins. The ankle, hindfoot, and forefoot were manually placed in 24 different ankle and foot positions. A stepwise regression analysis was used to examine the relationship among ankle, hindfoot, and forefoot kinematics and PT muscle excursion. Results: Hindfoot eversion/inversion and forefoot abduction/adduction accounted for $77 \%$ of the variance in PT muscle excursion, with small contributions from ankle plantarflexion/dorsiflexion $(5.7 \%)$ and forefoot plantarflexion/dorsiflexion (1.9\%). A combined regression equation applied to individual specimens resulted in average errors of less than $2.5 \mathrm{~mm}$. Conclusions: This study supports the hypothesis that PT muscle excursion can be estimated using specific foot and ankle kinematic variables. Further, these data suggest that hindfoot eversion and forefoot abduction account for most of the variance in PT muscle excursion and are theorized to be important to control clinically altering the length of the posterior tibial muscle.
\end{abstract}

\footnotetext{
${ }^{1}$ University of Rochester Medical Center, Rochester, NY

${ }^{2}$ University of Rochester, School of Nursing, Rochester NY

${ }^{3}$ Ithaca College Movement Analysis Laboratory, Ithaca College-Rochester Campus, Rochester, NY

Corresponding Author:

Jeff Houck, P.T., Ph.D.

Ithaca College - Rochester Campus

1100 South Goodman St.

Rochester, NY 14620

Email: jhouck@ithaca.edu

For information on prices and availability of reprints, call 410-494-4994 X226
}

Key Words: Cadaver study; Foot position; Posterior Tibial Tendon Muscle Excursion

\section{INTRODUCTION}

The posterior tibial muscle-tendon complex plays a significant role in controlling hindfoot inversion and forefoot adduction. $^{16}$ Adult acquired flatfoot deformity (AAFD) secondary to posterior tibial tendon dysfunction (PTTD) is characterized by varying degrees of increased forefoot abduction and hindfoot valgus. ${ }^{15,22}$ This increased forefoot abduction at the mid-tarsal joint and hindfoot eversion at the subtalar joint are hypothesized to further increase posterior tibialis (PT) muscle length leading to decreased function. ${ }^{9}$ Abnormal alignment associated with AAFD may increase PT muscle length, stretching the tendon, and shifting the force/length curve of the muscle contributing to weakening of the muscle. ${ }^{25,26}$ Nonoperative treatment of PTTD attempts to maintain normal alignment to protect foot ligaments and to improve PT muscle function. ${ }^{1,2,4,28}$ Braces and orthotic devices used for PTTD are designed to support the medial longitudinal arch (MLA) and decrease hindfoot eversion but rarely correct the forefoot abduction known to occur in subjects with PTTD. ${ }^{2,4,23,27}$

A positive relationship between hindfoot eversion and forefoot abduction and increased PT muscle length is assumed; however, the relative contributions of these foot kinematics to PT muscle length has not been determined. Posterior tibial muscle length is a function of the moment arm and the range of motion of each joint it crosses. ${ }^{16,17}$ Because the PT muscle crosses several joints, modeling muscle length through anatomic reconstruction of the moment arm of each joint is difficult. ${ }^{16}$ The PT muscle has the largest moment arm for inversion at the subtalar joint, ${ }^{9,13}$ yet no studies document the moment arms of the PT muscle across the joints that contribute to the forefoot abduction observed in subjects with PTTD. Further, applying in vitro data to walking data requires a better understanding of the relative contributions 
of different foot kinematic variables to changes in PT muscle length.

Establishing the precise relationship among foot kinematic variables and PT muscle length may provide a method to predict PT muscle length in vivo. ${ }^{8,11}$ Recent studies have established techniques for in vivo tracking of foot kinematics using skin-mounted markers. ${ }^{3,12,14,29}$ Yet, the validity of drawing inferences about structures internal to the foot, such as PT muscle length, using foot kinematic variables remains untested. Because changes in muscle length across a gait cycle are expected to be small, ${ }^{21}$ the errors associated with predicting muscle length using foot kinematics should be assessed. A positive relationship between ankle and foot kinematic variables and PT muscle length may lead to useful in vivo estimates of PT muscle length.

The purpose of this study was to examine the effect of hindfoot position in the frontal plane (eversion/inversion), forefoot position in the transverse plane (abduction/adduction), and ankle position in the sagittal plane (plantarflexion/dorsiflexion) on PT muscle excursion using a cadaver model. The PT muscle excursion was assumed to be directly associated with PT muscle length. Each kinematic variable was hypothesized to positively contribute to the variance of posterior tibial muscle excursion. In addition, the partial correlations for each kinematic variable were hypothesized to scale with their respective moment arms. For example, the moment arm is known to be larger for hindfoot eversion and inversion than ankle plantarflexion and dorsiflexion, therefore the partial correlation was expected to be larger for hindfoot eversion and inversion than for ankle plantarflexion and dorsiflexion. The moment arm for forefoot abduction and adduction is poorly understood; however, we hypothesized that the partial correlations would lie between hindfoot eversion and inversion and ankle plantarflexion and dorsiflexion.

\section{MATERIALS AND METHODS}

\section{Cadaver Preparation}

Seven fresh frozen cadaver specimens of the tibia and foot were thawed overnight until they were at room temperature. The average age of the cadaver specimens was 78.1 (range 67 to 79) years. Each cadaver specimen was prepared by sawing through the proximal tibia, potting the tibia in cement, and mounting the potted tibia on a platform (Figure 1). The PT tendon was dissected proximal to the medial malleoli, preserving the flexor retinaculum. A $5-\mathrm{kg}$ weight was sutured to the PT tendon using a $\mathrm{O}$ Ethibond (Ethicon, Inc, Piscataway, NJ, USA) in an interlacing fashion approximately $15 \mathrm{~cm}$ proximal to the medial malleoli. After ensuring that the tendon moved freely with foot movement, threaded 5/64 inch $\times 9$ inch bone pins were inserted into the tibia, calcaneus, and first metatarsal bones. Each bone pin was checked manually to ensure that the bone pin resisted twisting motions and bending due to soft-tissue strain. The platform was aligned so that vertical displacement of an infrared emitting diode (IREDs) placed on the 5-kg weight tracked PT muscle excursion.

\section{Instrumentation}

A six-camera Optotrak Motion Analysis System (Northern Digital Inc, CAN) was used to track three infrared emitting diodes mounted on each bone pin using custom made

\section{In-Vitro Specimen Testing Apparatus}

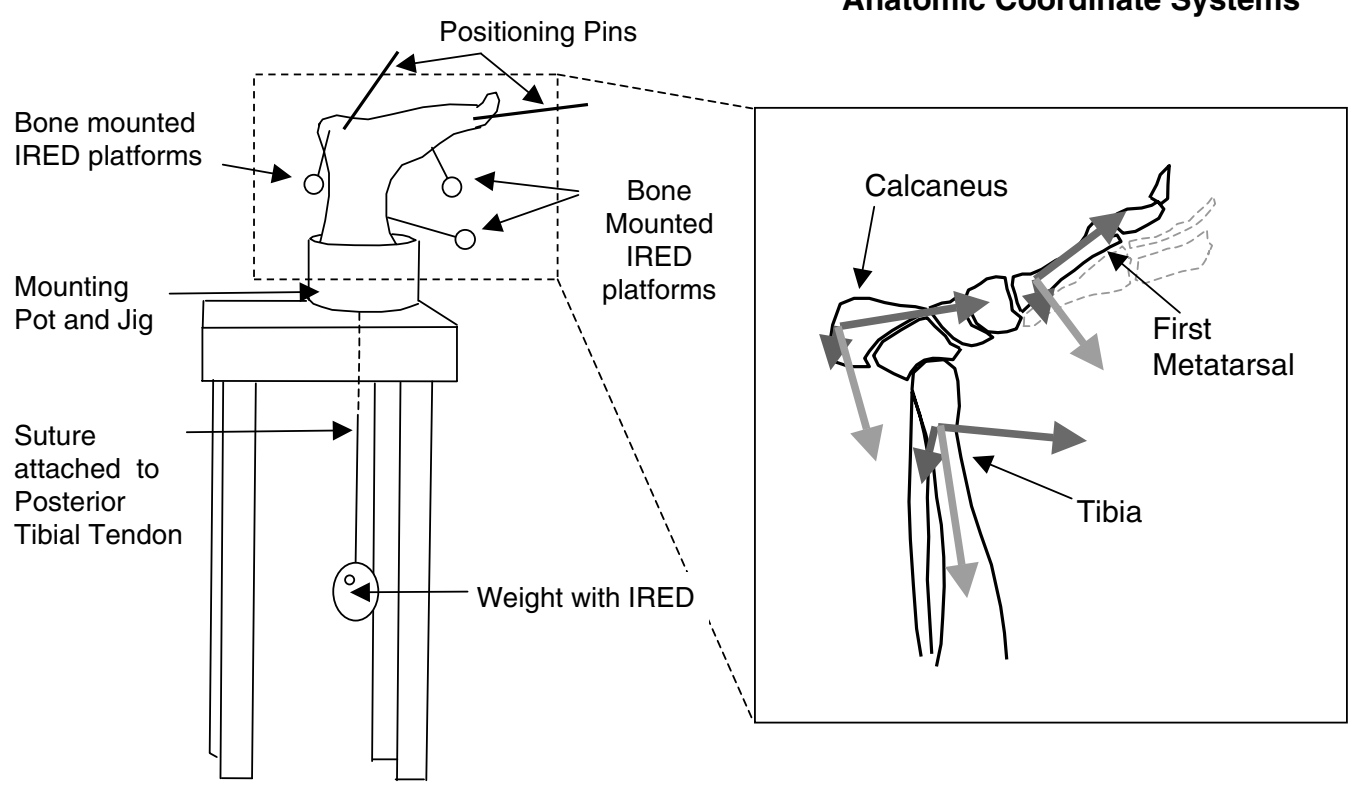

Kinematic Foot Model with Anatomic Coordinate Systems

Fig. 1: In-Vitro Specimen Testing Apparatus Kinematic Foot Model with Anatomic Coordinate Systems. 
platforms. The platforms were rigidly attached to the bone pins with threaded bolts (Figure 2). Each set of three IREDs allowed for rigid body representation of bone movement (Figure 1). The manufacturer reports an accuracy of tracking markers within $\pm<1 \mathrm{~mm}$ and $\pm<1$ degrees.

\section{Kinematic Modeling}

Using Motion Monitor Software (Innsport Training Inc, USA) online feedback of 3-D angles (Z-X' -Y" sequence) was used to achieve reproducible 3-D forefoot and hindfoot positions (Figure 1). The Optotrak Motion Analysis System is integrated with Motion Monitor Software (Innsport Training Inc, USA), allowing online tracking of rigid body representation of each bone. Kinematic data were smoothed using a fourth order, zero phase lag, Butterworth filter with a cut off frequency of $6 \mathrm{~Hz}$. Three digitized bony landmarks were used to establish anatomic coordinate systems for each segment. A right-handed Cartesian frame was used to represent each segment. The three digitized points used to establish a Cartesian frame for the calcaneus were a midpoint on the posterior and lateral heel, peroneal trochlea, and sustentaculum tali. The three digitized points used to establish a Cartesian frame for the first metatarsal were the base, lateral aspect of the

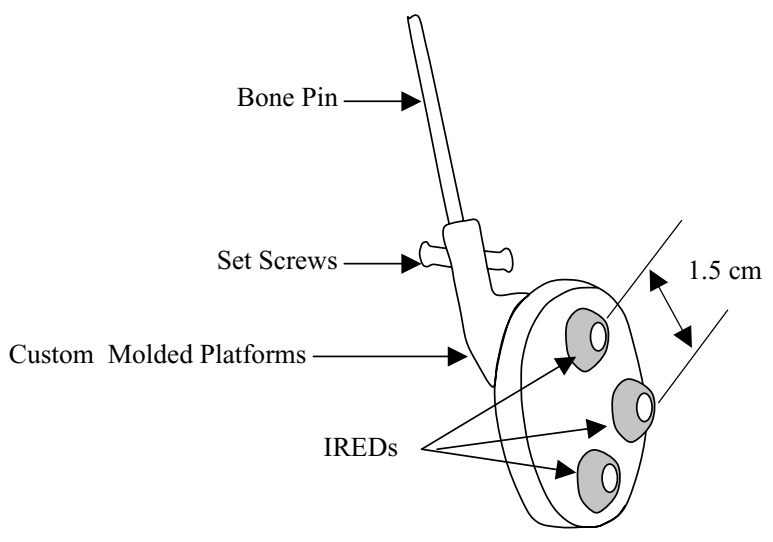

Fig. 2: Bone Mounted IRED platforms head, and medial aspect of the head of the metatarsal. The three digitized points used to establish a Cartesian frame for the tibia were the distal most point on the fibula, lateral malleous, and medial malleolus. Once a Cartesian frame was established for each segment (Figure 1), relative angles were calculated for the first metatarsal with respect to the calcaneus (forefoot position) and calcaneus relative to the tibia (hindfoot position). As recommended by others, ${ }^{5}$ a Z-X'Y" Cardan sequence was used to establish 3-D angles for each position. The kinematic variables calculated included 1) hindfoot eversion and inversion, 2) forefoot abduction and adduction, 3) ankle plantarflexion and dorsiflexion, and 4) forefoot plantarflexion and dorsiflexion.

\section{Procedures}

The goal was to use on-line feedback of 3-D angles to position the hindfoot and forefoot to achieve repeatable target positions to estimate PT muscle excursion. Forefoot motion was stabilized and controlled manually by inserting a separate bone pin into the calcaneus and first metatarsal. The first metatarsophalangeal joint was plantarflexed to allow the bone pin to be inserted into the shaft of the first metatarsal. This had the desired effect of relaxing the flexor hallucis muscles (brevis and longus) and the plantar fascia associated with the first metatarsophalangeal joint. The first metatarsal bone pin was subsequently used to manually move the forefoot into abduction and adduction (first metatarsal relative to the calcaneus), while maintaining a relatively constant internal and external and plantarflexion and dorsiflexion position. Hindfoot motion was manually stabilized and controlled by inserting a bone pin into the calcaneus and manipulating the position into eversion and inversion (calcaneus relative to the tibia), while maintaining relatively constant internal and external and plantarflexion and dorsiflexion positions. The actual target positions achieved for hindfoot eversion and inversion, forefoot abduction and adduction, and ankle plantarflexion and dorsiflexion are listed in Tables 1 and 2.

Table 1: Mean angles for targeted ankle, hindfoot and forefoot positions (mean SD)

\begin{tabular}{lcccc}
\hline \multicolumn{4}{c}{ Ankle } \\
\cline { 3 - 5 } & & DF & Neutral & PF \\
\hline Hind foot & EV & $-4.2 \pm 2.5$ degrees & $-5.3 \pm 1.8$ degrees & $-1.4 \pm 3.0$ degrees \\
& Neutral & $-0.8 \pm 3.1$ degrees & $0.7 \pm 2.1$ degrees & $2.4 \pm 2.6$ degrees \\
& INV & $8.1 \pm 2.6$ degrees & $9.6 \pm 2.2$ degrees & $12.0 \pm 3.5$ degrees \\
Fore foot & ABD & $-13.8 \pm 5.9$ degrees & $-13.2 \pm 4.0$ degrees & $-12.8 \pm 6.8$ degrees \\
& Neutral & $-1.2 \pm 3.6$ degrees & $-0.4 \pm 1.3$ degrees & $-0.0 \pm 3.6$ degrees \\
& ADD & $9.8 \pm 5.8$ degrees & $8.0 \pm 3.4$ degrees & $8.6 \pm 4.3$ degrees \\
\hline
\end{tabular}

Eversion, EV; Inversion, INV; Abduction, ABD; Adduction, ADD.

Negative values indicate hindfoot eversion and forefoot abduction. 
Table 2: Mean (SD) angles for targeted ankle positions with the hindfoot and forefoot in neutral

\begin{tabular}{llll}
\hline & \multicolumn{3}{c}{ Ankle } \\
\cline { 2 - 4 } & \multicolumn{1}{c}{ DF } & Neutral & PF \\
\hline HF \& FF Neutral & $10.8 \pm 2.6$ degrees & $0.1 \pm 1.9$ degrees & $-31.8 \pm 2.7$ degrees \\
\hline $\begin{array}{l}\text { Hindfoot, HF; Forefoot, FF; Dorsiflexion, DF; Plantarflexion, PF. } \\
\text { Negative values indicate ankle plantarflexion. }\end{array}$ & \\
\hline
\end{tabular}

Table 3: Mean $( \pm \mathrm{SD})$ of posterior tibial muscle excursion $(-=$ shortened, $+=$ lengthened $)$ for each position in millimeters

\begin{tabular}{|c|c|c|c|c|c|c|c|c|}
\hline & \multicolumn{2}{|c|}{$\begin{array}{c}\text { Combined foot } \\
\text { positions (HF EV) }\end{array}$} & \multicolumn{4}{|c|}{ Isolated foot positions } & \multicolumn{2}{|c|}{$\begin{array}{c}\text { Combined foot } \\
\text { positions (HF INV) }\end{array}$} \\
\hline & $\begin{array}{c}\text { HF EV + } \\
\text { FF ABD }\end{array}$ & $\begin{array}{c}\text { HF EV + } \\
\text { FF ADD }\end{array}$ & FF ABD & HF EV & HF INV & FF ADD & $\begin{array}{c}\text { HF INV + } \\
\text { FF ABD }\end{array}$ & $\begin{array}{c}\text { HF INV + } \\
\text { FF ADD }\end{array}$ \\
\hline \multicolumn{9}{|l|}{ Ankle } \\
\hline Dorsiflexion & $8.3 \pm 3.1$ & $3.1 \pm 3.3$ & $6.7 \pm 1.5$ & $3.1 \pm 2.5$ & $-1.1 \pm 2.0$ & $-2.8 \pm 2.6$ & $4.1 \pm 2.3$ & $-4.3 \pm 2.9$ \\
\hline Neutral & $7.2 \pm 2.1$ & $0.9 \pm 2.2$ & $5.2 \pm 1.4$ & $3.5 \pm 1.3$ & $-3.0 \pm 1.6$ & $-3.6 \pm 2.6$ & $2.8 \pm 2.6$ & $-6.3 \pm 2.0$ \\
\hline Plantarflexion & $2.8 \pm 2.0$ & $-3.0 \pm 4.0$ & $1.2 \pm 4.1$ & $-0.4 \pm 2.8$ & $-5.6 \pm 2.3$ & $-6.0 \pm 2.3$ & $-0.6 \pm 1.6$ & $-9.2 \pm 1.9$ \\
\hline
\end{tabular}

Each specimen was conditioned by moving it through plantarflexion and dorsiflexion of the ankle, eversion and inversion of the hindfoot, and abduction and adduction of the forefoot several times before testing. The forefoot (first metatarsal) and hindfoot (calcaneus) were placed in subtalar neutral established by palpation of the talus and navicular bones, while holding the calcaneus in neutral plantarflexion and dorsiflexion. This subtalar neutral position was used as an estimate of resting length for the PT muscle ( $\left.\mathrm{L}_{\mathrm{PTO}}\right)$. The reliability of the subtalar neutral position was tested with each cadaver three times throughout the testing protocol. The average difference (average of the absolute value [difference between trials]) among the three subtalar neutral trials was $0.8 \mathrm{~mm}$ across all specimens, suggesting that the examiner was able to reliably reproduce this neutral position. In addition, because these neutral trials occurred three times, intermittently, throughout the sequence of testing, they suggested that the effects of tendon creep were less than $0.8 \mathrm{~mm}$ on average. From this subtalar neutral position, the foot was moved into the 24 targeted hindfoot eversion and inversion and forefoot abduction and adduction positions and ankle plantarflexion and dorsiflexion positions (Tables 1 and 2). Once the online feedback from the Motion Monitor Software indicated the targeted position was achieved, the PT muscle excursion $\left(\mathrm{L}_{\mathrm{PT}}\right)$ was recorded. This process was repeated for each target position. The change in PT muscle excursion $\left(\Delta \mathrm{L}_{\mathrm{PT}}\right)$ was used in the analysis: $\Delta \mathrm{L}_{\mathrm{PT}}=\mathrm{L}_{\mathrm{PTO}}$ $\mathrm{L}_{\mathrm{PT}}$

\section{Analysis}

To further examine the relationship between the change in PT muscle excursion (dependent variable) and the specified kinematic variables (independent variables), a stepwise multiple regression analysis was used. The specified kinematic variables included those manipulated in the experiment: forefoot abduction and adduction and hindfoot eversion and inversion and ankle plantarflexion and dorsiflexion. Forefoot plantarflexion and dorsiflexion, which was not manipulated as part of the experiment, also was included to account for any inconsistencies in positioning. A stepwise multiple regression analysis using SPSS v13.0 software (SPSS, Inc., Chicago, IL, USA) using the F test with an alpha of less than 0.05 to determine inclusion of each variable in the model was performed. To assess whether a typical relationship existed across specimens or whether there was variability in the linear relationship across specimens, the regression analysis was first performed on each individual specimen's data (24 test positions) and secondarily on all the data across specimens ( 24 test positions $\times$ seven specimens). To assess the consequence of applying the combined 
Table 4: Results of regression analysis of all angle data considered together $(24$ positions per specimen $\times 7$ specimens)

\begin{tabular}{|c|c|c|c|c|c|c|c|c|}
\hline \multirow[b]{2}{*}{ Model } & \multirow[b]{2}{*}{$r^{2}$} & \multirow[b]{2}{*}{ Adj. $r^{2}$} & \multirow[b]{2}{*}{ SEE $( \pm \mathbf{m m})$} & \multicolumn{5}{|c|}{ Change Statistics } \\
\hline & & & & $R^{2}$ Change & F Change & df1 & df2 & Sig. F Change \\
\hline HF EV/INV & 0.445 & 0.442 & 3.7 & 0.445 & 148.4 & 1 & 185 & $<.001$ \\
\hline $\begin{array}{l}\text { HF EV/INV + FF } \\
\text { ABD/ADD }\end{array}$ & 0.770 & 0.767 & 2.4 & 0.325 & 259.6 & 1 & 184 & $<.001$ \\
\hline $\begin{array}{l}\text { HF EV/INV + FF } \\
\text { ABD/ADD + HF } \\
\text { PF/DF }\end{array}$ & 0.827 & 0.824 & 2.1 & 0.057 & 60.1 & 1 & 183 & $<.001$ \\
\hline $\begin{array}{c}\text { HF EV/INV + FF } \\
\text { ABD/ADD + HF } \\
\text { PF/DF + FF PF/DF }\end{array}$ & 0.846 & 0.843 & 2.0 & 0.019 & 23.1 & 1 & 182 & $<.001$ \\
\hline
\end{tabular}

HF EV/INV, hindfoot eversion/inversion; FF ABD/ADD, forefoot abduction/adduction; HF PF/DF, hindfoot plantarflexion/dorsiflexion; FF PF/DF, forefoot plantarflexion/dorsiflexion; SEE, standard error of the estimate; df, degrees of freedom; Sig., significant.

Table 5: Coefficients for each independent variable and $r^{2}$ (standard error of the estimate (SEE) in $\mathrm{mm}$ ) values for each specimen individually and combined

\begin{tabular}{lccccc}
\hline Specimen & HF EV/INV & FF ABD/ADD & HF PF/DF & FF PF/DF & $\boldsymbol{r}^{\mathbf{2}(S E E)}$ \\
\hline 1 & 0.627 & 0.300 & $\mathrm{NS}$ & 0.037 & $0.96(1.2 \mathrm{~mm})$ \\
2 & 0.601 & 0.341 & 0.008 & 0.010 & $0.95(1.2 \mathrm{~mm})$ \\
3 & 0.202 & 0.470 & 0.299 & $\mathrm{NS}$ & $0.97(0.8 \mathrm{~mm})$ \\
4 & 0.255 & 0.609 & 0.105 & $\mathrm{NS}$ & $0.97(0.8 \mathrm{~mm})$ \\
5 & 0.333 & 0.041 & 0.014 & 0.578 & $0.96(1.0 \mathrm{~mm})$ \\
6 & 0.485 & 0.301 & 0.073 & $\mathrm{NS}$ & $0.84(1.7 \mathrm{~mm})$ \\
7 & 0.528 & 0.358 & 0.019 & $\mathrm{NS}$ & $0.89(1.7 \mathrm{~mm})$ \\
& & All specimen considered together & & \\
Combined & 0.447 & 0.311 & 0.055 & 0.021 & $0.85(2.0 \mathrm{~mm})$ \\
\hline
\end{tabular}

HF EV/INV, hindfoot inversion/eversion; FF ABD/ADD, forefoot abduction/adduction; HF PF/DF, hindfoot plantarflexion/dorsiflexion; FF PF/DF, forefoot plantarflexion/dorsiflexion.

regression equation calculated across specimens to an individual, the $r^{2}$ and root mean square (rms) difference between the measured change in PT muscle excursion $\left(\Delta \mathrm{L}_{\mathrm{PT}}\right)$ and predicted change in PT muscle excursion using the combined regression equation was calculated. The advantage of using a regression analysis is that it accounts for differences between specimens in the target position achieved. For example, when performing the combined hindfoot and forefoot positions it was not always possible to achieve the exact target position (See Hindfoot Eversion + Ankle Plantarflexion, Table 1). Because the regression analysis tests whether there is a linear relationship among variables, irrespective of the target positions achieved, small differences across specimens in the target position have little influence on the results. Further, using extreme hindfoot and forefoot positions increases the range of the independent variables, enhancing the ability to statistically detect linear relationships.

\section{RESULTS}

\section{Regression Analysis of Individual Specimens}

For each individual specimen, the independent variables retained in the regression equation always included hindfoot eversion and inversion and forefoot abduction and adduction but not hindfoot or forefoot plantarflexion and dorsiflexion (Table 4). For one of the seven specimens hindfoot plantarflexion and dorsiflexion were not significant, and for four of the seven specimens forefoot plantarflexion and dorsiflexion were not significant, so these variables were not included in the individual specimen regression equation 
(Table 4). For all the specimens, the $r^{2}$ values were above 0.84 and the standard error of the estimates below $1.7 \mathrm{~mm}$. Five of the seven specimens had $r^{2}$ values above 0.9 and standard errors of the estimates of below $1.2 \mathrm{~mm}$.

\section{Regression Analysis of All Specimens Combined}

Across specimens hindfoot eversion and inversion and forefoot abduction and adduction influenced PT muscle excursion more than the other independent variables (Table 3 ). Hindfoot eversion and inversion entered the equation first with an $r^{2}$ of $0.445(\mathrm{~F}(1,185)=148.4, p<0.001)$, followed by the forefoot abduction and adduction with an $r^{2}$ change of $0.325(\mathrm{~F}(1,184)=259.6, p<0.001)$. Ankle position (hindfoot plantarflexion and dorsiflexion), with an $r^{2}$ change of $0.057(\mathrm{~F}(1,183)=60.1, p<0.001)$ entered the equation next followed by forefoot plantarflexion and dorsiflexion $(\mathrm{F}(1,182)=23.1, p<0.001)$ which resulted in an $r^{2}$ change of 0.019 . This model accounted for $85 \%$ $\left(r^{2}=0.846\right)$ of the variance in PT muscle excursion with a standard error of the estimate of $2.0 \mathrm{~mm}$. The coefficients for each independent variable are reported in the equation below and in Table 5.

$$
\begin{aligned}
& \text { Predicted } \Delta \mathrm{L}_{\mathrm{PT}}=0.76+0.401(\mathrm{HF} \text { Ev } / \mathrm{Inv}) \\
& +0.270(\mathrm{FF} \text { Abd/Add })+0.057(\mathrm{HF} \text { Pf } / \mathrm{Df}) \\
& +0.137(\mathrm{FF} \text { Pf } / \mathrm{Df}) \pm 2.0 \mathrm{~mm}
\end{aligned}
$$

The $r^{2}$ values ranged from 0.82-0.94 when comparing the measured $\Delta \mathrm{L}_{\mathrm{PT}}$ to the predicted $\Delta \mathrm{L}_{\mathrm{PT}}$ using the equation (Table 6). The range of rms differences for each specimen was 1.6 to $2.5 \mathrm{~mm}$.

\section{DISCUSSION}

The findings of this study suggest that hindfoot eversion and inversion and forefoot abduction and adduction have a greater influence on PT muscle excursion than forefoot plantarflexion and dorsiflexion and ankle plantarflexion and dorsiflexion. Consistent across all specimens was the strong influence of hindfoot eversion and inversion and forefoot abduction and adduction on PT muscle excursion. The combined regression analysis suggests that hindfoot eversion and inversion alone explains $45 \%$ of the variance in PT muscle excursion. The most consistent pattern across specimens was for the hindfoot eversion and inversion and forefoot abduction and adduction to account for most of the variance in PT muscle excursion $\left(r^{2}=0.77\right)$. Although hindfoot eversion and forefoot abduction are known to be key components of the deformity associated with PTTD, the differential effect of these positions on muscle excursion have not been previously described. The findings of this study suggest a significant role of forefoot abduction and hindfoot
Table 6: The $r^{2}$ and root mean square error between measured change in posterior tibial muscle excursion and estimated posterior tibial muscle excursion using the combined regression equation applied to each specimen

\begin{tabular}{llc}
\hline Specimen & $\boldsymbol{r}^{\mathbf{2}}$ & rms error $(\mathbf{\pm m m})$ \\
\hline 1 & 0.90 & 2.2 \\
2 & 0.83 & 1.8 \\
3 & 0.83 & 2 \\
4 & 0.93 & 1.8 \\
5 & 0.82 & 2.5 \\
6 & 0.92 & 1.6 \\
7 & 0.94 & 1.6 \\
\hline
\end{tabular}

eversion on increasing excursion of the PT muscle-tendon unit.

Muscle excursion is a function of the moment arm and excursion of the joint the muscle crosses. ${ }^{16,17}$ Previous studies suggested that the PT muscle has an approximately $1.5-\mathrm{cm}$ muscle excursion for hindfoot eversion and inversion over a range of -9 to 21 (30 degrees). ${ }^{9}$ The changes in PT muscle excursion observed in the neutral ankle position for hindfoot eversion (HF EV) and inversion (HF INV) (Table 3) in this study (HF EV-HF INV = $3.5-(-3.5 \mathrm{~mm})=6.5 \mathrm{~mm})$ are similar if the smaller range of motion (14.9 degrees $=\mathrm{HF} \mathrm{EV}-\mathrm{HF} \mathrm{INV}=-5.3$ degrees-9.6 degrees) is taken into account (Table 1). The PT muscle excursion from the maximally shortened position of ankle plantarflexion with hindfoot inversion combined and forefoot adduction (Table 3, HF INV + FF ADD, Ankle Plantarflexion $=-9.2 \mathrm{~mm}$ ), to a maximally lengthened position of ankle dorsiflexion with hindfoot eversion and forefoot abduction (Table 3, HF EV + FF ABD, Ankle Dorsiflexion $=8.3 \mathrm{~mm}$ ), suggested an average $1.75 \mathrm{~cm}$ $(17.5 \mathrm{~mm}=8.3 \mathrm{~mm}+9.3 \mathrm{~mm})$ PT muscle excursion. The similarities across studies are interesting given no axial load or tendon forces, other than the PT muscle, were simulated in this study. Because the PT muscle has the largest moment arm for hindfoot eversion and inversion and the shortest moment arm for ankle plantarflexion and dorsiflexion, ${ }^{9}$ hindfoot position was expected to be the primary contributor to PT muscle excursion. What is new to this study is the significant contribution of forefoot abduction and adduction to PT muscle excursion.

Unique to this study, the change in PT muscle excursion associated with forefoot positions suggested a large moment arm of the forefoot around an abduction and adduction joint axis. The range of movement in forefoot abduction and adduction was approximately 20 degrees (Table 1) and the PT muscle excursion was $8.8 \mathrm{~mm}$ (Table 3), suggesting a moment arm of $2.5 \mathrm{~cm}(25 \mathrm{~mm}=$ 
$8.8 \mathrm{~mm} /[20$ degrees $/ 57.3$ degrees $])$. This moment arm estimate is smaller than the average for hindfoot eversion and inversion of 3 to $4 \mathrm{~cm}$ calculated from previous studies; however, it is larger than the moment arm for plantarflexion and dorsiflexion. ${ }^{9,13}$ What is unknown is the effect of second through fifth metatarsal movement and which mid-tarsal and metatarsal joints (talonavicular, naviculocuneiform, cuneiform metatarsal) contributed to PT muscle excursion. Given that the PT muscle does not attach to the first metatarsal, inclusion of the second through fourth metatarsals to move the forefoot into abduction and adduction may have led to different results. Alternatively, forefoot abduction and adduction may contribute to PT muscle excursion due to stretch of the attachment on the navicular bone. Given the kinematic modeling used in this study, we are unable to define which specific joints contributed to increasing PT muscle excursion during the forefoot abduction and adduction movements.

The high $r^{2}$ values suggest that the identified kinematic variables may be useful in estimating PT muscle excursion, and therefore PT muscle length, when combined with motion analysis techniques. The known measurement error of skin-mounted markers on the calcaneus and first metatarsal during simulated walking movements is low, ${ }^{18,24}$ suggesting that skin-mounted markers may yield relationships similar to those found in this study. Because the kinematic foot modeling used in this study is similar to recent clinical trials of subjects with PTTD walking, ${ }^{23}$ the combined regression equation developed in this study is potentially useful for estimating changes in PT muscle length. However, the individual regression equations resulted in higher $r^{2}$ values than the combined regression equation (Table 5), suggesting a loss of accuracy (approximately 10\%) when using the combined regression equation for individuals. The effect of applying the combined regression equation to individual specimens is reflected in the rms differences of less than $2.5 \mathrm{~mm}$ when the combined regression equation was applied to each specimen (Table 6). Because 2.5-mm errors may account for a high percentage of total excursion (38.4\% error for hindfoot eversion and inversion $=2.5 \mathrm{~mm} / 6.5 \mathrm{~mm} \times 100)$, movements expected to result in high amplitudes of muscle excursion are advisable to reduce the signal to noise ratio (3.3\% error for maximal excursion $=2.5 \mathrm{~mm} / 17.5 \mathrm{~mm} \times 100$ ). Also, when abnormal foot kinematics result in changes in PT muscle length of more than $2.5 \mathrm{~mm}$ the regression equation described in this study may be useful for comparing PT muscle excursion in vivo. However, to advance this application further, understanding why interspecimen variability occurs and how foot deformity affects these estimates is worthy of further investigation.

Various ankle braces, longitudinal arch supports, insole orthotic devices, and ankle-foot orthoses have been used in the nonoperative treatment of PTTD. In order to be effective, these devices may shorten the length of the posterior tibial tendon and supporting ligamentous structures by correcting foot alignment and controlling foot motion. Based on the findings of this study, forefoot abduction deformity contributes significantly to PT muscle length and, therefore, should be considered during treatment. Longitudinal arch supports with medial postings are designed to support the medial longitudinal arch and improve hindfoot eversion, and ankle braces frequently are used to control hindfoot inversion and eversion. ${ }^{7}$ These devices may not improve or control forefoot abduction. Ankle-foot orthoses that provide lateral forefoot posting are expected to be more effective in controlling abduction; however, their ability to do so has not been studied. Imhauser et al. ${ }^{10}$ performed a kinematic study to evaluate the ability of a variety of orthotic devices to stabilize the medial longitudinal arch and hindfoot in a simulated flatfoot model, but this study did not consider forefoot abduction. Operative approaches, such as lateral column lengthening, that also focus on improved hindfoot and forefoot alignment may shorten the PT muscle, inducing a therapeutic effect. ${ }^{19}$ However, this study suggests that further studies are needed that differentiate the effects of treatments (braces or orthotics and surgery) on control of hindfoot and forefoot motion and the length of the PT musculotendon complex.

Because direct measurements of PT muscle length in vivo are difficult, an in vitro model of PT muscle excursion was developed. Because the PT muscle crosses only the fibula-tibia syndesomosis proximal to the ankle joint, a direct relationship between PT muscle excursion attributable to foot and ankle kinematics and PT muscle length was assumed. Nonweightbearing positions were used because of the desire to generate a variety of foot positions, resulting in 24 different combinations of ankle, forefoot, and hindfoot position for each specimen. The results of this study are dependent on the linear relationship between muscle excursion and ankle and foot kinematics reported here not being affected by weightbearing status. The foot kinematic model also may have been more extensive, accounting for the contribution of other midfoot or forefoot joints to PT muscle excursion. However, the purpose of this study was to relate these particular kinematic foot variables to an in vivo technique constraining the research design. ${ }^{23}$ Future studies may consider more direct measures of muscle length and tendon strain using in vitro simulations of walking ${ }^{20}$ or musculotendon modeling that are able to take into account the dynamic aspect of muscle contractions. ${ }^{6}$

This study supports the hypothesis that PT muscle excursion can be estimated using specific foot and ankle kinematic variables. Further, these data suggest that hindfoot eversion and forefoot abduction account for most of the variance in PT muscle excursion and, therefore, are theorized to be important to control clinically to alter the length of the PT muscle. Optimal treatments (brace or orthotic design and surgery) to prevent progression of PTTD should include components to control both hindfoot and forefoot position. In addition, the regression equation listed may be used to model PT muscle 
length in vivo when combined with similar foot kinematic modeling.

\section{REFERENCES}

1. Alvarez, RG; Marini, A; Schmitt, C; Saltzman, CL: Stage I and II posterior tibial tendon dysfunction treated by a structured nonoperative management protocol: an orthosis and exercise program. Foot Ankle Int. 27:2-8, 2006.

2. Augustin, JF; Lin, SS; Berberian, WS; Johnson, JE: Nonoperative treatment of adult acquired flat foot with the Arizona brace. Foot Ankle Clin. 8:491-502, 2003.

3. Carson, MC; Harrington, ME; Thompson, N; O'Connor, JJ; Theologis, TN: Kinematic analysis of a multi-segment foot model for research and clinical applications: a repeatability analysis. J Biomech. 34:1299-307, 2001.

4. Chao, W; Wapner, KL; Lee, TH; Adams, J; Hecht, PJ: Nonoperative management of posterior tibial tendon dysfunction. Foot Ankle Int. 17:736-741, 1996.

5. Cole, GK; Nigg, BM; Ronsky, JL; Yeadon, MR: Application of the joint coordinate system to three-dimensional joint attitude and movement representation: a standardization proposal. J. Biomech. Eng. 115: 344-349, 1993.

6. Delp, SL; Loan, JP; Hoy, MG; et al.: An interactive graphics-based model of the lower extremity to study orthopaedic surgical procedures. IEEE Trans. Biomed. Eng. 37:757-767, 1990.

7. Elftman, NW: Nonsurgical treatment of adult acquired flat foot deformity. Foot Ankle Clin. 8:473-489, 2003.

8. Herzog, W; Read, LJ: Lines of action and moment arms of the major force-carrying structures crossing the human knee joint. J. Anat. 182:213-230, 1993.

9. Hintermann, B; Nigg, BM; Sommer, C: Foot movement and tendon excursion: an in vitro study. Foot Ankle Int. 15: 386-395, 1994

10. Imhauser, CW; Abidi, NA; Frankel, DZ; Gavin, K; Siegler, S: Biomechanical evaluation of the efficacy of external stabilizers in the conservative treatment of acquired flatfoot deformity. Foot Ankle Int. 23:727-737, 2002

11. Kepple, TM; Arnold, AS; Stanhope, SJ; Siegel, KL: Assessment of a method to estimate muscle attachments from surface landmarks: a 3D computer graphics approach. J. Biomech. 27:365-371, 1994.

12. Kidder, SM; Abuzzahab, FS, Jr.; Harris, GF; Johnson, JE: A system for the analysis of foot and ankle kinematics during gait. IEEE Trans. Rehabil. Eng. 4:25-32, 1996.

13. Klein, P; Mattys, S; Rooze, M: Moment arm length variations of selected muscles acting on talocrural and subtalar joints during movement: an in vitro study. J. Biomech. 29:21-30, 1996.
14. Leardini, A; Benedetti, MG; Catani, F; Simoncini, L; Giannini, S: An anatomically based protocol for the description of foot segment kinematics during gait. Clin. Biomech. 14: 528-536, 1999.

15. Niki, H; Ching, RP; Kiser, P; Sangeorzan, BJ: The effect of posterior tibial tendon dysfunction on hindfoot kinematics. Foot Ankle Int. 22:292-300, 2001

16. Otis, JC; Gage, T: Function of the posterior tibial tendon muscle. Foot Ankle Clin. 6:1-14, 2001.

17. Pandy, MG: Moment arm of a muscle force. Exerc. Sport Sci. Rev. 27:79-118, 1999.

18. Reinschmidt, C; van den Bogert, AJ; Lundberg, A; et al.: Tibiofemoral and tibiocalcaneal motion during walking: external vs. skeletal markers. Gait Posture 6:98-109, 1997.

19. Sangeorzan, BJ; Mosca, V; Hansen, ST, Jr.: Effect of calcaneal lengthening on relationships among the hindfoot, midfoot, and forefoot. Foot Ankle. 14:136-141, 1993.

20. Sharkey, NA: One step at a time: lessons learned from cadaver simulations of locomotion. J. Orthop. Sports Phys. Ther. 34:A7-9, 2004.

21. Sutherland, DH: An electromyographic study of the plantar flexors of the ankle in normal walking on the level. J. Bone Joint Surg. 48A:66-71, 1966.

22. Thordarson, DB; Schmotzer, H; Chon, J; Peters, J: Dynamic support of the human longitudinal arch. A biomechanical evaluation. Clin. Orthop. 316:65-72, 1995

23. Tome, J; Flemister, S; Nawoczenski, D; Houck, J: Comparison of foot kinematics between subjects with posterior tibial tendon dysfunction and healthy controls. J. Orthop. Sports Phys. Ther. 36:635-344, 2006.

24. Umberger, BR; Nawoczenski, DA; Baumhauer, JF: Reliability and validity of first metatarsophalangeal joint orientation measured with an electromagnetic tracking device. Clin. Biomech. 14:74-76, 1999.

25. Valderrabano, V; Hintermann, B; Wischer, T; Fuhr, P; Dick, W: Recovery of the posterior tibial muscle after late reconstruction following tendon rupture. Foot Ankle Int. 25:85-95, 2004.

26. Wacker, J; Calder, JD; Engstrom, CM; Saxby, TS: MR morphometry of posterior tibialis muscle in adult acquired flat foot. Foot Ankle Int. 24: 354-357, 2003.

27. Wapner, KL,Chao, W: Nonoperative treatment of posterior tibial tendon dysfunction. Clin. Orthop. 365:39-45, 1999.

28. Williams, R: Chronic Non-Specific tendovaginitis of the tibialis posterior. J. Bone Joint Surg. 45-B: 542-545, 1963.

29. Woodburn, J; Nelson, KM; Siegel, KL; Kepple, TM; Gerber, LH: Multisegment foot motion during gait: proof of concept in rheumatoid arthritis. J. Rheumatol. 31:1918-1927, 2004. 